\title{
Spin dynamics in liquid and rotationally disordered solid oxygen
}

\author{
F. Fernandez-Alonso, ${ }^{1, *}$ F. J. Bermejo, ${ }^{2, \dagger}$ I. Bustinduy, ${ }^{2}$ M. A. Adams, ${ }^{1}$ and J. W. Taylor ${ }^{1}$ \\ ${ }^{1}$ ISIS Facility, Rutherford Appleton Laboratory, Chilton, Didcot, Oxfordshire OX11 0QX, United Kingdom \\ ${ }^{2}$ Instituto de Estructura de la Materia, C.S.I.C., and Departamento de Electricidad y Electrónica - Unidad Asociada C.S.I.C., Facultad \\ de Ciencia y Tecnología, Universidad del País Vasco/EHU, P.O. Box 644, E-48080 Bilbao, Spain
}

(Received 13 June 2008; revised manuscript received 19 August 2008; published 15 September 2008)

\begin{abstract}
The spin and nuclear dynamics of the liquid and plastic-solid phases of molecular oxygen are studied by means of inelastic neutron scattering. A detailed spectral analysis over wide energy- $(0-18 \mathrm{meV})$ and momentum- $\left(0.2-4.5 \AA^{-1}\right)$ transfer ranges permits a robust separation of magnetic and nuclear contributions to the dynamic structure factor. Reliable spin-exchange constants are obtained for both liquid and plastic phases, which are found to be remarkably similar to each other, with no evidence for long-range antiferromagnetic spin correlations beyond next-nearest neighbors. Analysis of the time scales involved in spin-flip processes suggests that spin diffusion in these structurally disordered phases takes place within a lapse of time comparable to the lifetime of an $\mathrm{O}_{4}$ unit in the liquid.
\end{abstract}

DOI: 10.1103/PhysRevB.78.104303

PACS number(s): 61.05.fg, 75.50.Mm, 61.25.Em, 61.43.-j

\section{INTRODUCTION}

Because of its exceptionally strong paramagnetism, liquid oxygen (LOX) exhibits several remarkable macroscopic properties if subjected to a magnetic field. ${ }^{1}$ These go from changes in liquid volume and color ${ }^{2}$ under applied field to levitation and pattern formation phenomena at the liquid surface, ${ }^{1}$ some of which have a clear technological relevance. Macroscopic magnetic phenomena resulting from the paramagnetic nature of the liquid such as the Faraday rotation of microwaves have been known for quite some time, ${ }^{3}$ although a truly microscopic understanding of the underlying physical phenomena has not been attempted. The molecular origin of such a strong paramagnetism is easily understood on qualitative grounds as resulting from Hund's rule, whereby two antibonding electrons occupy $\pi_{2 p}^{*}$ molecular orbitals resulting in a triplet $(S=1)$ electronic ground state for the isolated oxygen molecule $\left(\mathrm{O}_{2}\right) .{ }^{4}$ However, a quantitative assessment of the relative importance of the magnetic versus nuclear interactions as well as their interplay remains to be established.

The presence within the liquid of significant short-range magnetic correlations has been unequivocally established from detailed polarized neutron-diffraction studies. ${ }^{5}$ The scattered intensity of magnetic origin displays a broad maximum centered at about $1.2 \AA^{-1}$ with a hydrodynamic limit given by

$$
I(Q \rightarrow 0)=3 k_{B} T \chi(T) / S(S+1)\left(g \mu_{B}\right)^{2},
$$

where $S=1$ and $g=2$. Setting the paramagnetic susceptibility $\chi(T)$ to its experimental value, ${ }^{6}$ one gets an average magnetic moment per atom $\mu_{\text {eff }} \approx 0.8 \mu_{B}$, substantially below the freemolecule value $g \sqrt{S(S+1)}=2.83 \mu_{B}$. Both polarized neutron diffraction and computer simulation ${ }^{7}$ suggest that oxygen molecules form dimer structures with lifetimes as long as 0.6 ps, where the molecular axes are confined within parallel planes separated by some $3.6 \AA$. Such an arrangement of molecules is also a dominant structural motif in the rotatorphase (plastic) crystal which forms by cooling the liquid below $54.4 \mathrm{~K}$ at ambient pressure. ${ }^{8}$ Freezing the liquid into the rotationally disordered crystal known as $\gamma-\mathrm{O}_{2}(\mathrm{GOX})$ is mediated by an anomalously small drop in molar volume, together with a meager $3.5 \%$ increase in susceptibility, and yields a cubic unit-cell structure of symmetry $P m 3 n$, an A15 structure with eight molecules per cell. One fourth of these molecules sit at the corners of the cubic cell ( $2 a$ sites) and exhibit complete (isotropic) molecular reorientations ${ }^{9}$ as well as a spherically symmetric electron-density distribution, whereas the rest sit at the cube faces ( $6 d$ sites), forming one-dimensional (1D) chains characterized by an oblateshaped electron distribution. Molecules in these chains rotate within disk-shaped volumes at right angles to the $\langle 100\rangle$ crystal axis. Explicit calculations show that such a structure is mostly stabilized by the isotropic part of the intermolecular potential $^{8}$ while the action of anisotropic interactions is almost entirely felt by the disks. The magnetic structure of $\gamma-\mathrm{O}_{2}$ also resembles that of the liquid, showing a broad diffuse peak in the single-differential magnetic cross section for polarized neutrons $s^{5,9}$ centered at about $1.0 \AA^{-1}$. As far as the strength of the magnetic interactions is concerned, the observation of diffraction peaks of diffuse nature dictates all subsequent data analysis to be carried out in terms of model fits. A value for the magnetic moment per molecule of $\mu_{\mathrm{eff}}$ $=2.65 \mu_{B}$ was derived from the best fit to an $X-Y$ model with spins perpendicular to the molecule axis. Magnetic correlations are of a predominant antiferromagnetic character and dominated by nearest neighbors separated by $3.37 \AA$. Such a value of $\mu_{\text {eff }}$ is, however, well above that determined from neutron diffraction ${ }^{10}$ for the magnetically ordered $\alpha$ phase at low temperatures corresponding to $\mu_{\mathrm{eff}}=1.45 \mu_{B}$.

Here we pursue the close analogy of the magnetic properties of LOX and GOX to obtain additional information on the strength and role of the magnetic interactions by means of an analysis of time-dependent properties known to be more sensitive to fine details of the spin dynamics than those of structural nature. Previous neutron scattering data reported some time ago $^{11}$ were restricted to a relatively narrow range of momentum and energy transfers leading to significant uncertainties in the values of the derived parameters. The crystalline GOX phase is here considered as a pertinent reference for the liquid in order to tackle and quantify mass-diffusion 
effects present in LOX. Also, and contrary to previous attempts ${ }^{11}$ which relied somewhat on model fits, the route here followed to analyze the experimental data does not rely on fits to model functions in order to isolate the magnetic component from the total neutron scattering spectra. Such a component, once isolated, is shown to provide estimates of the spin-diffusion constant and thus yields new information pertaining exchange constants and energy scales involved in spin motions for this important molecular material.

\section{EXPERIMENTS}

Experiments were performed using the MARI (Ref. 12) inelastic neutron spectrometer at the ISIS Pulsed Neutron and Muon Source, United Kingdom. An incident energy $E_{0}$ $=18 \mathrm{meV}$ was employed to map the dynamic structure factor $S(Q, \omega)$ over momentum- and energy-transfer ranges of $0.2-4.5 \AA^{-1}$ and $\pm 16 \mathrm{meV}$, respectively. The resolution in energy transfer was of $\Delta E=0.3 \mathrm{meV}$ full width at half maximum (FWHM). Neutron spectra were corrected for detector efficiency using a vanadium standard followed by subtraction of the contribution from the stainless-steel empty cell. An annular geometry of $1 \mathrm{~cm}$ corresponding to a total scattering power of $\sim 5 \%$ was used to minimize absorption and multiple-scattering effects. Temperature control was achieved by the use of a closed-cycle helium refrigerator. High-purity $\mathrm{O}_{2}$ gas (CK gases, $99.9 \%$ purity) was condensed from a room-temperature reservoir into the sample cell held at $T=88 \mathrm{~K}$ and subsequently cooled down to the LOX $(T$ $=65 \mathrm{~K})$ and $\operatorname{GOX}(T=50 \mathrm{~K})$ phases. Temperature stability during the experimental runs was within $1 \mathrm{~K}$.

Once corrected for background and other instrumental effects, measured intensities were mapped onto a $Q-\omega$ grid. As shown in Fig. 1, images were then reconstructed from the measured data by means of a purpose-built algorithm, ${ }^{13}$ which avoids introducing substantial correlation between adjacent bins.

\section{RESULTS}

Owing to its zero nuclear spin $(I=0)$ and subsequent lack of a permanent nuclear magnetic moment, the predominant nuclide in naturally occurring oxygen $\left({ }^{16} \mathrm{O}, 99.76 \%\right)$ is a purely coherent neutron scatterer with a nuclear scattering cross-section $\sigma_{\text {nuc }}=4.232$ barn per nuclide and a negligible absorption cross section $\left(\sigma_{\text {abs }} \sim 2 \times 10^{-4}\right.$ barn at thermalneutron wavelengths). In addition to the above nuclear contribution, the $\mathrm{O}_{2}$ molecule in its ${ }^{3} \Sigma_{g}^{-}$ground electronic state has a permanent electronic spin $S=1$ giving rise to a paramagnetic differential cross-section $d \sigma_{\text {mag }} / d \Omega=0.389 f^{2}(Q)$ barn/steradian, where $f(Q)$ is the magnetic form factor for the isolated molecule. ${ }^{14}$ From these preliminary considerations one may conclude that both correlated nuclear motions leading to coherent scattering as well as spin motions due to the magnetic nature of the $\mathrm{O}_{2}$ molecule will give contributions to the neutron spectra, and their relative weight is dependent on the range of explored wave vectors.

\section{A. Bayesian spectral analysis}

The information content of the inelastic neutron data shown in Fig. 1 has been assessed by means of a line shape
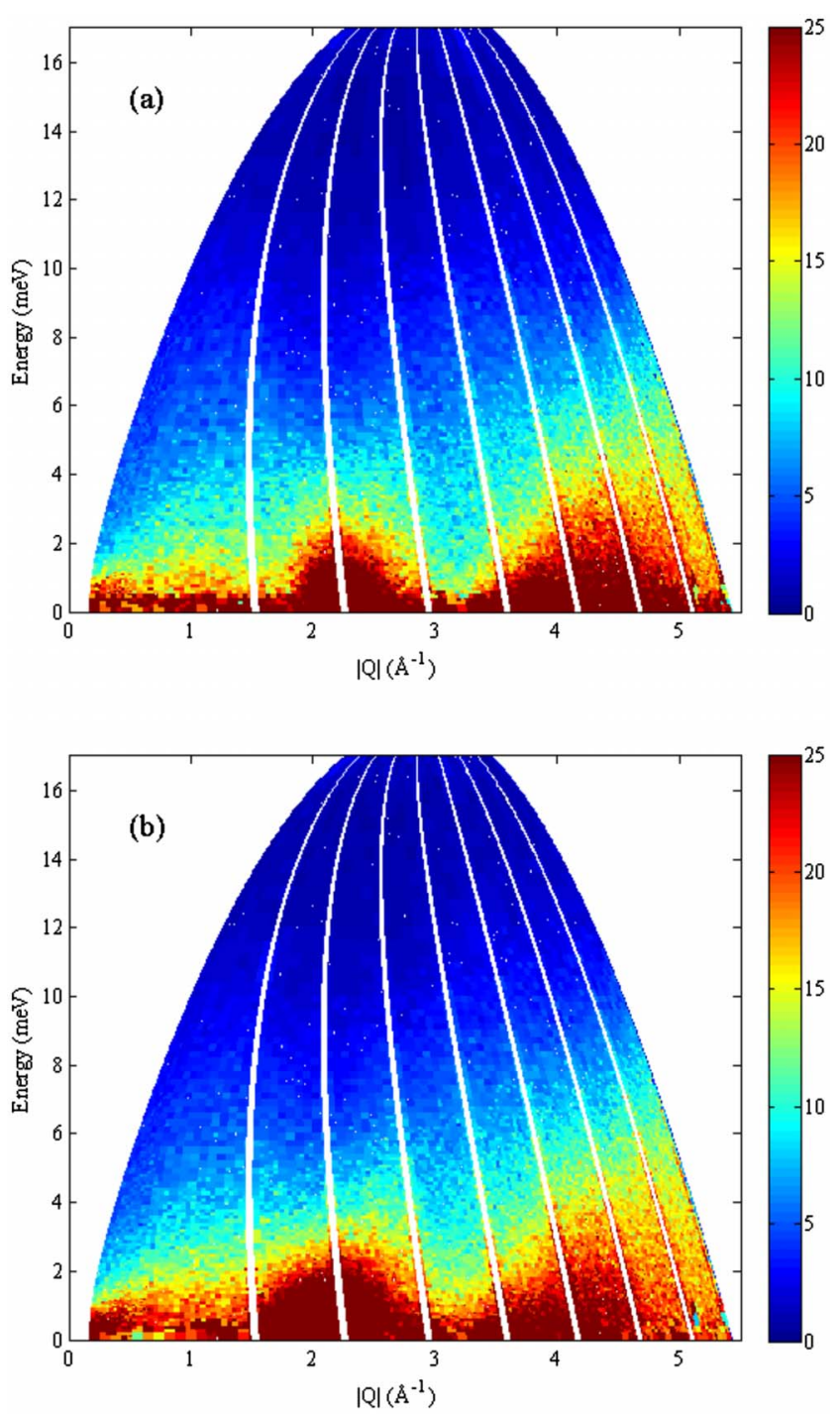

FIG. 1. (Color online) $\mathrm{O}_{2}$ neutron spectra measured on MARI at an incident energy of $E_{0}=18 \mathrm{meV}$. (a) Gamma phase $(T=50 \mathrm{~K})$ and (b) liquid $(T=65 \mathrm{~K})$.

analysis. To this end, a Bayesian algorithm was used to find the highest number of Lorentzian modes supported by the experimental data ${ }^{15}$ using a dynamic structure factor $S(Q, \omega)$ of the form

$$
S(Q, \omega)=R(Q, \omega) \otimes\left[A_{0} \delta(\omega)+\sum_{i=1}^{N} \frac{A_{i}}{\pi} \frac{\Gamma_{i} / 2}{\omega^{2}+\left(\Gamma_{i} / 2\right)^{2}}\right],
$$

where $R(Q, \omega)$ is the instrumental resolution function and $\otimes$ is a convolution product. $A_{i}$ denotes the integrated intensity of elastic $(i=0)$ or quasielastic $(i>0)$ components, and $\Gamma_{i}$ is the Lorentzian energy width (FWHM) for the $i$ th quasielastic mode. To use Eq. (2), the experimental data have been symmetrized via a detailed-balance factor of the form $e^{-\hbar \omega / 2 k_{B} T}$. As shown in Fig. 2, our experimental data only justify the use of a single quasielastic mode for both GOX and LOX in 
$\gamma-\mathrm{O}_{2} \mathrm{~T}=50 \mathrm{~K}$
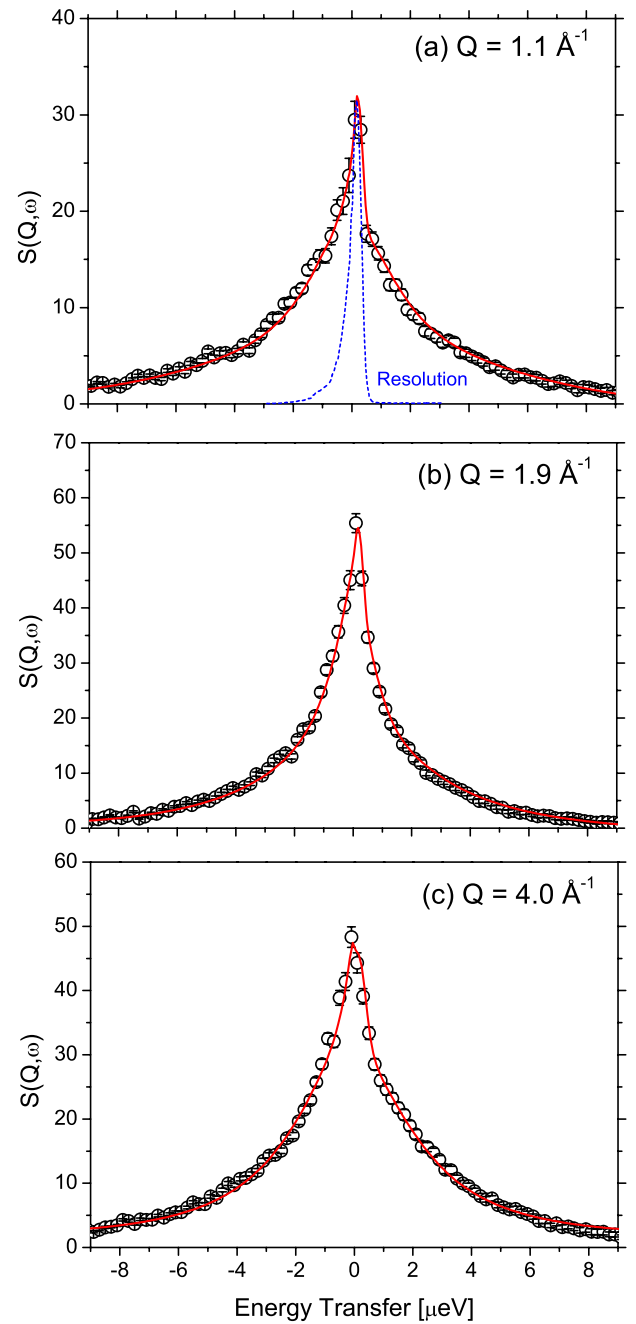

Liquid $\mathrm{O}_{2} \mathrm{~T}=65 \mathrm{~K}$
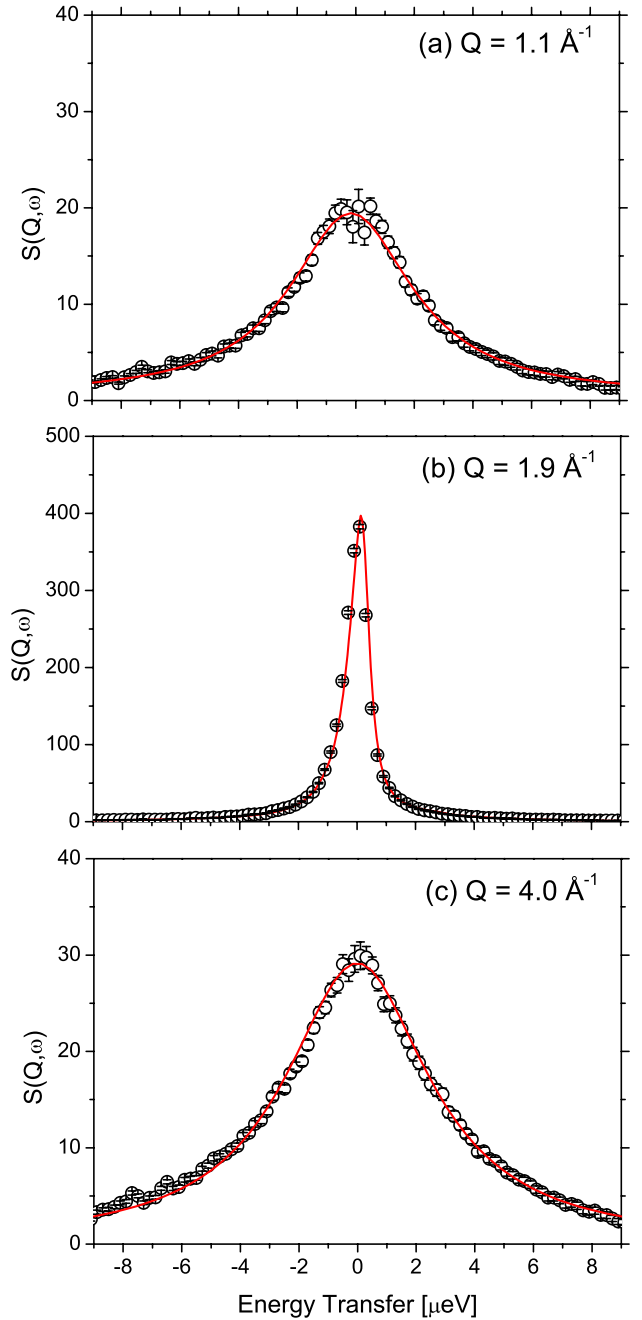

FIG. 2. (Color online) Inelastic neutron spectra at selected momentum transfers (open circles) and associated fits (solid red lines) for $N=1$ as described by Eq. (2) in the text. In all cases, the instrumental resolution (dashed blue line) has been folded into our Lorentzian model for the purposes of fitting. addition to an elastic component in GOX to account for static correlations in the plastic phase.

The results of such spectral analysis are displayed in Fig. 3. The momentum-transfer dependence of the quasielastic widths and intensities at low $\left(Q<1 \AA^{-1}\right)$ and high $(Q$ $>3 \AA^{-1}$ ) momentum transfers is remarkably similar in both phases. Significant differences are only observed at momentum transfers around the structural (elastic) peak in GOX at $2.1 \AA^{-1}$ which, upon melting, gives rise to strong and broad scattering over the explored $Q-\omega$ range. This behavior is indicative of very similar dynamical processes in GOX and LOX apart from translational diffusion of the molecular center of mass in the latter case.

\section{B. Spectral frequency moments}

On qualitative grounds, the neutron data for the liquid and gamma phases shown in Figs. 1 and 2 display remarkably close patterns. Prominent spectral features are present in the dynamic structure factor at wave vectors $Q \sim 2$ and $4 \AA^{-1}$. As expected for a cubic crystal such as GOX, these features are sharper in $Q$ for the gamma phase, but it is to be noted that characteristic spectral widths extend up to $5-6 \mathrm{meV}$ in both cases, a finding which is suggestive of a common dynamical origin. Also, there is a significant amount of scattering extending to low-momentum transfers, peaking at roughly $Q \sim 1 \AA^{-1}$, that is, roughly half the momentum transfer where the main peak of the nuclear structure factor is located in both phases. Such broad features provide a first model-free assessment of the relative weight of the magnetic singly differential cross section versus that arising from atomic correlations.

In order to put the above remarks on more quantitative grounds, Fig. 4 shows the integrated intensity and reduced second $\left\langle\omega^{2}\right\rangle^{1 / 2}$ and fourth $\left\langle\omega^{4}\right\rangle^{1 / 4}$ frequency moments for both liquid and gamma $\mathrm{O}_{2}$. These quantities have been evaluated using discrete integrations of the measured spectral functions according to

$$
\left\langle\omega^{n}\right\rangle=\int \omega^{n} S(Q, \omega) d \omega / \int S(Q, \omega) d \omega .
$$

The total integrated intensity shows main peaks arising from the static structure factors $S(Q)$ centered in both cases at $Q_{\max } \sim 2.1 \AA^{-1}$, together with broad features located roughly at $Q_{\max } / 2$. On the other hand, the second spectral frequency 

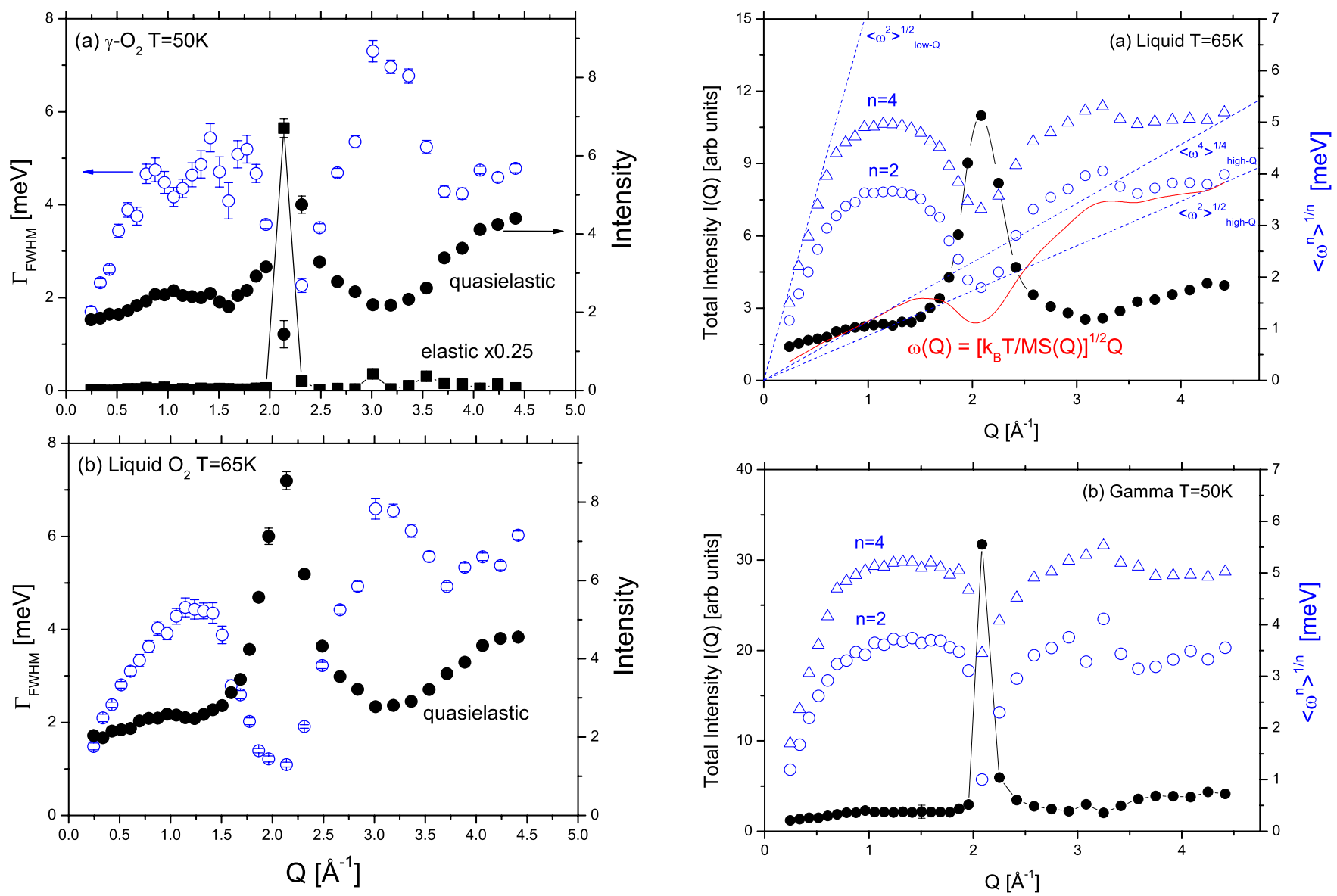

FIG. 3. (Color online) Integrated intensities as defined by Eq. (2) (right $y$ axis; solid black circles) and quasielastic widths (left $y$ axis; open blue circles) obtained from the Bayesian analysis described in the text.

moment shows for both LOX and GOX rather similar features, displaying a quasilinear $Q$ dependence up to about $0.7 \AA^{-1}$ and a maximum reaching some $3.5 \mathrm{meV}$ at $Q \sim 1.2 \AA^{-1}$ followed by a minimum, which coincides with the position of the maxima in $S(Q)$. Since the second frequency moment can be analytically calculated for a simple liquid, the data for the liquid phase [cf. Fig. 4(a)] can be compared with the idealgas limit for such a quantity given by $\left\langle\omega^{2}\right\rangle^{1 / 2}=v Q$, where $v$ $=\sqrt{k_{B} T / M}=0.868 \AA \mathrm{meV}$, a quantity which our experimental data approach at high-momentum transfers. The same applies to the reduced fourth frequency moment which approaches $3^{1 / 4} Q \sqrt{k_{B} T / M}$ at large momentum-transfer values. Further, the $Q$ dependence of the coherent linewidths is also compared to the expectation for nuclear scattering from a structureless liquid given by $\left\langle\omega^{2}\right\rangle^{1 / 2}=Q \sqrt{k_{B} T / M S(Q)}$. The same considerations would apply if such a spectral width is calculated using more refined estimates based on kinetic theory arguments, which are known to reproduce the coherent linewidth in simple liquids. ${ }^{16}$

The comparison between experimental data shown in Fig. 4(a) and the two estimates for the linewidths referred to above shows that the observed spectral widths for the liquid for wave vectors below $\sim 1.5 \AA^{-1}$ cannot be sensibly assigned as arising from the nuclear dynamics. In fact, as expected from knowledge of many other liquids, the free-gas

FIG. 4. (Color online) Total integrated intensities (left axis, black symbols) and reduced second and fourth spectral moments (right axis, blue symbols) for (a) liquid $(T=65 \mathrm{~K})$ and (b) gamma phase $(T=50 \mathrm{~K})$. Red (solid) and blue (dashed) lines correspond to the limits and approximations described in the text.

regime is approached for wave vectors beyond $4 \AA^{-1}$. Furthermore, data at the lowest $Q$ 's are also compared to the hydrodynamic dispersion law $\Omega_{\text {coll }}(Q)=c_{s} Q$, which is expected to be obeyed within such a range of momentum transfers by collective density oscillations propagating with phase velocity $c_{s}=7.24 \AA \mathrm{meV}$ corresponding to the macroscopic sound velocity. ${ }^{17,18}$ In other words, the comparison just referred to tells that the linewidths observed at low $Q$ 's cannot be assigned to overdamped density oscillations. These are expected to follow a quasilinear dependence of an average frequency defined in terms of the bare $\omega_{b}$ and damping $\Gamma$ terms as $\Omega_{\text {coll }}=\sqrt{\omega_{b}^{2}+\Gamma^{2}}$ which, as shown in the figure, lies consistently above the experimental data. Based on these considerations, neutron scattering in the region $Q<1.5 \AA^{-1}$ is assigned to the predominance of magnetic scattering in both LOX and GOX.

Additional confirmation of the dominant magnetic nature of the observed linewidths for $Q<1.5 \AA^{-1}$ is provided by a direct comparison of LOX and GOX data in Fig. 4. In this respect, one notices that the low- $Q$ slopes of $\left\langle\omega^{2}\right\rangle^{1 / 2}$ are rather close, whereas the average sound velocity increases by over $30 \%$. 
Given the above, we can now consider the GOX data on more quantitative grounds. For a polycrystal, where magnetic interactions are confined to $n$ first-nearest neigbors separated by a distance $d_{n n}$, the $Q$ dependence of the second frequency moment in the high-temperature limit can be expressed as ${ }^{19}$

$$
\hbar^{2}\left\langle\omega^{2}\right\rangle=\frac{8}{3} n S(S+1) J^{2}\left[1-\frac{\sin \left(Q d_{n n}\right)}{Q d_{n n}}\right],
$$

from where estimates of a single (average) exchange constant $J$ can be derived provided that the number of nearest neighbors is known. The data for GOX can only be fitted using Eq. (4) if the distance $d_{n n}$ is left as a free parameter. The ensuing value for such a quantity yields $d_{n n}$ $=3.91 \pm 0.09 \AA$, which is well above that of $d_{n n}=3.37 \AA$ known from careful diffraction studies on single crystals ${ }^{9}$ and corresponds to nearest neighbors in a chain of disklike molecules, which are expected to experience the strongest magnetic interactions. To overcome such difficulty, we have set the value for the shortest distance to its experimental estimate of $d_{n n}=3.37 \AA$ and that for the number of nearest neigbors $n=2$. This choice then requires to include an additional interaction by adding a second term to Eq. (4) so that it becomes

$$
\begin{aligned}
\hbar^{2}\left\langle\omega^{2}\right\rangle= & \frac{8}{3}\left\{n_{n n} J_{n n}^{2}\left[1-\frac{\sin \left(Q d_{n n}\right)}{Q d_{n n}}\right]\right. \\
& \left.+n_{n n n} J_{n n n}^{2}\left[1-\frac{\sin \left(Q d_{n n n}\right)}{Q d_{n n n}}\right]\right\},
\end{aligned}
$$

where the second term lumps together all magnetic interactions with next-nearest neighbors. The best fit was obtained leaving such a distance as an adjustable parameter and yields $d_{n n n}=5.9 \pm 0.3 \AA$, a distance which comprises the characteristic lengths of both disk-sphere, interchain, and intersphere interactions; the results of such fits are shown in Fig. 5. Magnetic exchange constants are derived by setting $n_{n n}=2$ and $n_{n n n}=12$, in agreement with the crystal structure, leading to $J_{n n}=0.84 \pm 0.03 \mathrm{meV}$ characterizing the strongest magnetic interactions as well as $J_{n n n}=0.11 \pm 0.07 \mathrm{meV}$, which measures the strength of exchange interactions beyond nearest neighbors. These values are to be compared with $J_{n n}$ $=1.22 \pm 2.0 \mathrm{meV}$ and $J_{n n n}=0.17-0.26 \mathrm{meV}$ obtained from high-field magnetization data. ${ }^{20}$

Data for LOX can also be fitted using the expression given above and a single exchange constant and yields values for both the characteristic distance $d_{n n}=4.01 \pm 0.05 \AA$ and that for the exchange parameter $J_{n n}=0.41 \pm 0.05 \mathrm{meV}$. This analysis assumes a value of $n_{n n}=12$ as given by integration of the radial distribution function for molecular centers up to the first minimum at $5.3 \AA,^{7}$ corresponding to the first shell of molecules. This value of $J_{n n}$ in LOX is close to $0.47 \mathrm{meV}$, the arithmetic average of nearest- and next-nearest-neighbor exchange constants in GOX. It is to be noted, however, that while the value for the characteristic distance is not far from that corresponding to the separation between molecular centers $d_{\mathrm{cm}} \sim 3.8 \AA$ in the liquid, the value used for the coordination number $n_{n n}$ largely exceeds that expected for the liq-

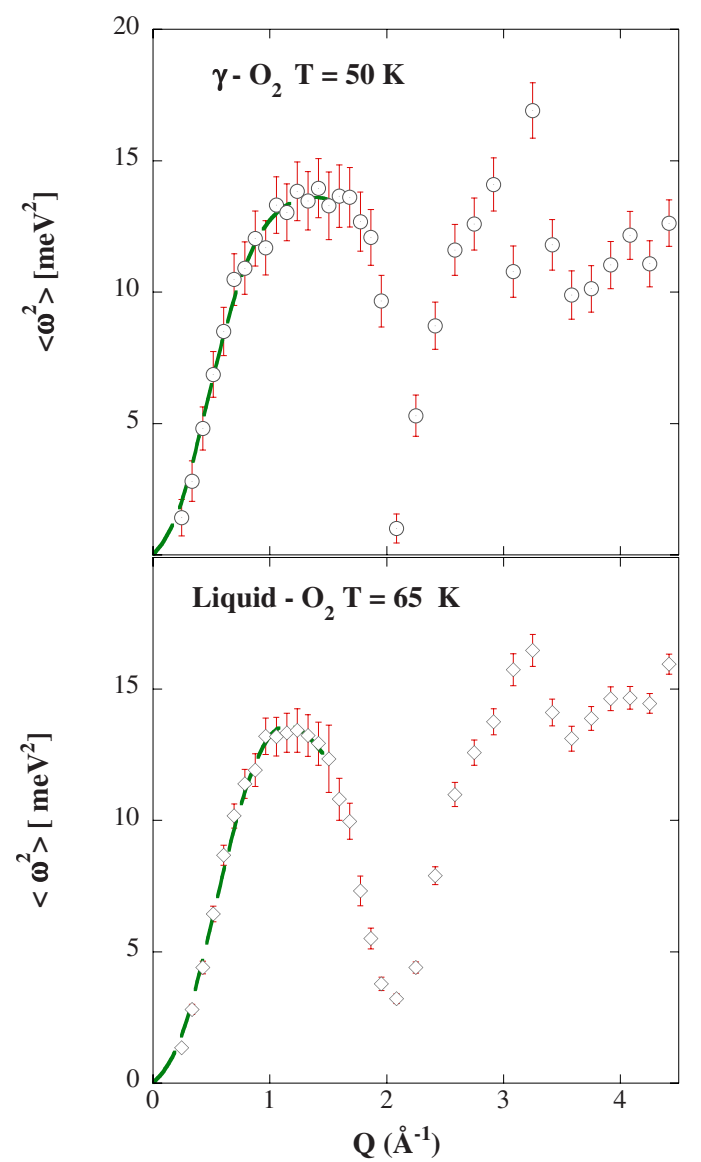

FIG. 5. (Color online) A comparison between the experimental values for the second frequency moments for GOX (upper panel symbols) and LOX (lower panel symbols) and fits given by Eqs. (4) and (5) (solid lines).

uid within a sphere of radius $r=4 \AA$, for which a coordination number about $n_{n n} \sim 5$ is expected. If this reduction in $n_{n n}$ is taken into account, a slightly stronger magnetic interaction characterized by $J_{n n}=0.64 \pm 0.1 \mathrm{meV}$ is then obtained. From these considerations, we conclude that our two estimates for $J_{n n}$ represent conservative upper and lower limits to the average nearest-neighbor magnetic exchange constant in LOX. Most importantly, magnetic interactions in LOX are comparable to those found in GOX, further illustrating the remarkable similarities between these two phases.

\section{Timescales for spin diffusion}

For both GOX and LOX, experimental data below $Q$ $=0.7 \AA^{-1}$ show that spectral widths follow a linear dependence $\Gamma(Q)=D_{m} Q$ with a coefficient $D_{m} \sim 5 \mathrm{meV} \AA$. This defines a distance-time scale of $0.14 \AA \mathrm{ps}^{-1}$. As shown below, this figure is an order of magnitude faster than the characteristic translational ${ }^{21}$ and rotational ${ }^{22}$ motions measured in the liquid.

Experimental values for the translational diffusion coefficient are already available from macroscopic tracer measurements $^{21}$ that yield values of $0.15 \AA^{2} \mathrm{ps}^{-1}$ at $T$ $=77 \mathrm{~K}$. Estimates for the rotational diffusion constant $D_{R}$ may be obtained from the spectral widths shown in Fig. 3 by 
considering the nuclear dynamic structure factor for an ensemble of homonuclear diatomic molecules in the weakly hindered rotational approximation. ${ }^{23}$ For the case of a purely coherent scatterer such as the oxygen atom, it reads

$$
\begin{aligned}
S_{\mathrm{coh}}(Q, \omega)= & j_{0}^{2}(Q R / 2) S_{\mathrm{coh}}^{\mathrm{cm}}(Q, \omega) \\
& +\sum_{l>0}^{\text {even }}(2 l+1) j_{l}^{2}(Q R / 2)\left[S_{l}(\omega) \otimes S_{\mathrm{inc}}^{\mathrm{cm}}(Q, \omega)\right],
\end{aligned}
$$

where $R=1.21 \AA$ is the $\mathrm{O}-\mathrm{O}$ bond length, $S_{\text {coh }}^{\mathrm{cm}}(Q, \omega)$ and $S_{\text {inc }}^{\mathrm{cm}}(Q, \omega)$ are the coherent and incoherent-scattering laws for the molecular centers, respectively, $j_{l}$ 's are spherical Bessel functions of order $l$, and $S_{l}(\omega)$ are rotational spectral functions of the form

$$
S_{l}(\omega)=\frac{1}{\pi} \frac{\Gamma_{l} / 2}{\omega^{2}+\left(\Gamma_{l} / 2\right)^{2}},
$$

where $\Gamma_{l}=2 l(l+1) D_{R}$ is a Lorentzian width (FWHM). Given the internuclear distance of the oxygen molecule, the $l=2$ term dominates the spectral response in the range $Q$ $=4-4.5 \AA^{-1}$ as $j_{0}^{2} / 5 j_{2}^{2}<0.02$ and $9 j_{4}^{2} / 5 j_{2}^{2}<0.07$. These considerations then lead to rotational diffusion constants of $0.38 \pm 0.02$ and $0.46 \pm 0.02 \mathrm{meV}$ for GOX at $T=50 \mathrm{~K}$ and LOX at $65 \mathrm{~K}$, respectively. The associated characteristic timescales $\tau_{R}=\hbar D_{R}^{-1}$ amount to $1.7 \pm 0.1$ and $1.4 \pm 0.1 \mathrm{ps}$, in good agreement with the simulation results of Ref. 11 and optical birefringence experiments ${ }^{22}$ for liquid oxygen. Moreover, the temperature dependence of these timescales is rather mild and approaches the characteristic $\sqrt{T}$ behavior expected for a decoupled degree of freedom devoid of activation energy barrier.

These values for rotational diffusion may also be compared with those obtained from the Debye-Stokes-Einstein (DSE) equation, known to be valid on semiquantitative grounds $^{24,25}$ and given by

$$
D_{R}=\frac{k_{B} T}{8 \pi \eta \sigma^{3}},
$$

where $\eta$ stands for the viscosity and $\sigma$ stands for the effective particle radius. Using recent accurate data for the viscosity $^{26}$ as well as the value for the radius at contact derived from the computer analysis of the radial distribution function $\sigma=2.3 \AA, D_{R}=6 \times 10^{-3} \mathrm{ps}^{-1}$ which would correspond to a reorientational correlation time $\tau_{R} \approx 170 \mathrm{ps,}$ about two orders of magnitude slower than our experimental values. The value of the rotational relaxation time could only be brought down to $\tau_{R} \approx 2$ ps if the effective diameter $\sigma$ is set to the value given by the molecule radius that is far too short since it corresponds to vanishing values of the radial distribution. This is a rather catastrophic failure of the DSE relation to provide a sensible (order of magnitude) estimate for rotational timescales and a vivid reminder of the ability of molecules to execute rapid reorientational motions in media of very large macroscopic viscosity.

The $Q$ dependence of the quasielastic linewidths is also found to depart significantly from the $\Gamma(Q)=D_{m} Q^{2}$ law expected on hydrodynamic, i.e., Fick's law, grounds for spin- diffusion processes in Heisenberg magnets. Breakdown of the hydrodynamic assumptions in classical 1D Heisenberg magnets has been reported a number of times, mostly from spin-dynamics simulations ${ }^{27}$ and has been attributed to the presence of long-time tails in the decay of spin-spin correlations. Such departures are also found to be more marked as the dimensionality of the magnetic system is reduced from three dimensional to 1D. From a theoretical standpoint, the problem of spin diffusion in 1D Heisenberg systems devoid of long-range order has been revisited several times. ${ }^{28} \mathrm{Sev}$ eral approximate expressions to estimate the spin-diffusion coefficient mostly within the high-temperature limits have been previously reported. ${ }^{28}$ The analysis leads to a factorization of the diffusion coefficient in terms of a coefficient dependent on geometric and susceptibility parameters $A(T)$ and an associated relaxation time $\tau_{s}(T)$; that is,

$$
D_{s}(T)=A(T) \tau_{s}(T),
$$

where $A(T)$ has a known high-temperature limiting value given by $A(T \rightarrow \infty)=\frac{2}{3} S(S+1) J_{n n}^{2} a^{2} \hbar^{-2}$ and $a$ stands for the lattice constant. In turn, the relaxation time can be evaluated from a relation between spectral moments as

$$
\tau_{s}=\lim _{Q \rightarrow 0} \frac{\sqrt{2 / \pi}}{\hbar} \frac{\left\langle\omega^{4}\right\rangle^{1 / 2}}{\left\langle\omega^{2}\right\rangle^{3 / 2}} .
$$

Using the expression given above as a recipe for the relaxation times associated with spin diffusion, we obtain from the ratio of the calculated frequency moments values $\tau_{s}$ $=0.53 \pm 0.06 \mathrm{ps}$ for GOX and $\tau_{s}=0.55 \pm 0.03 \mathrm{ps}$ for LOX. The results again provide a striking confirmation of the similitude of the spin dynamics in both liquid and plastic crystal phases of this material. These timescales can be supplemented by estimates for the diffusion coefficient derived from hydrodynamic theory and thus serve to set values for $A(T)$. Using expressions given in Ref. 29, whereby this transport coefficient is expressed in terms of nearest-neighbor parameters as

$$
D_{s}^{\text {hyd }} \approx 0.23 d_{n n}^{2} \sqrt{S(S+1)} \frac{2 J_{n n}}{\hbar},
$$

and using the values for $d_{n n}$ and $J_{n n}$ given above, we get an estimate for GOX yielding $D_{s}^{\text {hyd }}=6.21 \mathrm{meV}^{2}$, as well as values within $4.3 \leq D_{s}^{\text {hyd }} \leq 6.01 \mathrm{meV}^{2}$ for LOX, depending on the set of $\left(J_{n n}, d_{n n}\right)$ pairs used in the calculation.

The values for the spin-diffusion coefficients and the associated relaxation times given above serve to set a time scale for spin-flip processes beyond the hydrodynamic realm. In fact, the factorization of $D_{s}(T)$ in terms of a characteristic relaxation time $\tau_{s}$ and a factor $A(T)$ can be written in terms of following an expression valid for all wave vectors (i.e., well beyond the hydrodynamic regime) as ${ }^{30}$

$$
D_{s}=\frac{n \mu^{2} \tau_{s}}{4 M \chi},
$$

which is given in terms of the number density $n$, particle mass $M$, its magnetic moment $\mu$, and the static susceptibility $\chi$. From here, one arrives at the high-temperature limit for $\tau_{s}$ (Ref. 30) that reads, 

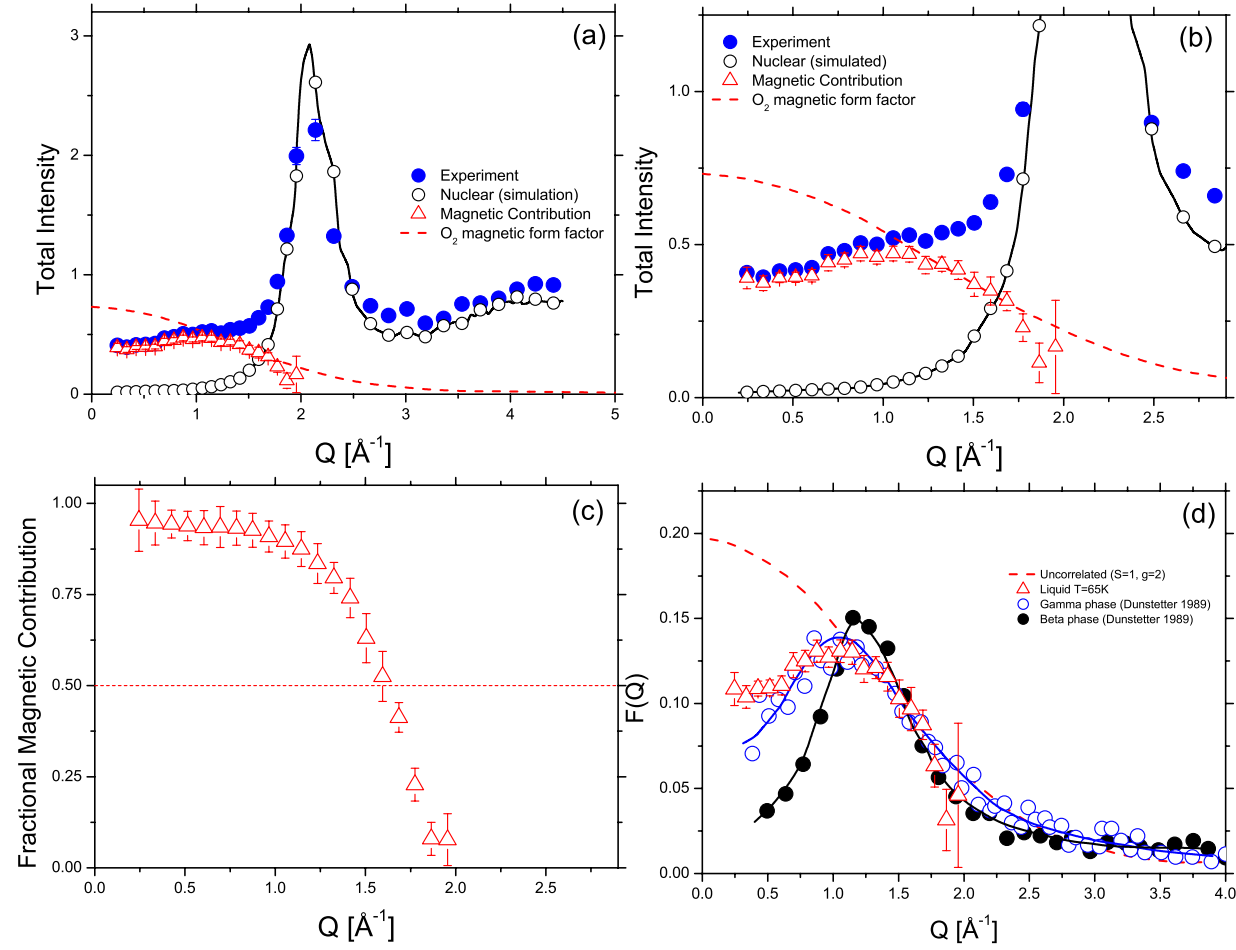

FIG. 6. (Color online) An assessment of the nuclear vs magnetic contributions to the LOX dynamic structure factor is shown in panels (a) and (b). (c) displays the fractional magnetic contribution as a function of momentum transfer. (d) compares the derived LOX magnetic structure factor with those for Gox and $\beta$ oxygen. For further details, see text.

$$
\tau_{s}(T \rightarrow \infty)=\sqrt{\frac{\pi}{2}} \hbar J_{n n}^{-1} \sqrt{\frac{6}{32 S(S+1)-3}} .
$$

Substitution of the relevant values within the above equation yields high-temperature estimates for the correlation time associated with spin diffusion of $0.4-0.63$ ps for LOX, depending on the value used for the exchange constants, and 0.3 ps for GOX.

\section{DISCUSSION}

At this point, it is worth assessing the robustness of the separation of magnetic and nuclear dynamics pursued in our previous analysis of experimental data.

Figure 6 compares the total integrated intensity as derived from our experimental LOX data with the static structure factor for structural (i.e., nuclear) correlations as calculated via first-principles computer simulation. ${ }^{7}$ Subtraction of these two data sets to obtain the magnetic contribution as a function of momentum transfer has been effected by finding the best subtraction factor (in the least-squares sense) that reproduces the magnetic form factor of an isolated oxygen molecule for $Q=1.5-2.0 \AA^{-1}$. This procedure is justified by earlier neutron diffraction on GOX, ${ }^{5}$ showing only significant deviations from the isolated-molecule limit below $Q$ $=1.0 \AA^{-1}$. Such a comparison serves to illustrate the dominant magnetic contribution to the structure factor for $Q$ values below $1.0 \AA^{-1}$, an explicit measure of which is given in Fig. 6(c).

Figure 6(d) puts things on an absolute scale and also provides a visual assessment of the strength of magnetic correlations within the liquid, GOX, as well as within the structurally ordered, albeit magnetically disordered, $\beta$ phase as derived from polarized neutron-diffraction data. ${ }^{5,9}$ As already remarked earlier on, spin correlations in LOX and GOX are astoundingly close, and the main difference found at low $Q$ 's is accounted for by the difference in magnetic susceptibilities. The correlation length of magnetic fluctuations as judged from the inverse peak widths $\eta=2 \pi / \Delta Q \approx 4.5 \AA$ becomes comparable in both rotationally disordered phases.

Let us now compare the estimates for the exchange constants here derived for the liquid with those calculated from magneto-optical and magnetovolume experiments carried out under high fields. ${ }^{20}$ These authors propose a distancedependent exchange constant derived from high-field data for $\alpha, \beta$, and $\gamma-\mathrm{O}_{2}$ phases which varies as ${ }^{20}$

$$
J(r)=J_{0} \exp \left[\varepsilon\left(r-r_{0}\right)\right],
$$

with $J_{0}=-2.63 \mathrm{meV}, \varepsilon=-4.3 \AA^{-1}$, and $r_{0}=3.2 \AA$. The values calculated using the formula given above for the characteristic distances, as derived from the analysis of the spectral frequency moments, yield a value for the exchange coupling within the liquid at a distance $d_{n n}=4.01 \AA$ of $0.081 \mathrm{meV}$, which is to be compared with $J_{n n}=0.41 \mathrm{meV}$ derived from Eq. (4) under the assumption of coupling to 12 nearest neighbors. It is to be noted that Eq. (4) enables the determination of the product $n_{n n} J_{n n}^{2}$ only and, therefore, the present data could only be reconciled with the macroscopic observation as given by Eq. (14) if the number of nearest neighbors coupled through such a low exchange constant increases up to a value not consistent with experimental data for the microscopic density function $d(r)=4 \pi \rho r^{2} g(r)$. However, both macroscopic and microscopic data can be reconciled if the distance to nearest neighbors is set to the value given by the position of the main peak in the radial distribution for molecular centers, $d_{n n}=3.8 \AA$, and further allowance is made for about $10 \%$ of the molecules to be coupled at a distance of $3.4 \AA$ by a stronger exchange constant of $1.2 \mathrm{meV}$. This 
latter result is thus suggestive of the presence of a sizeable fraction of strongly coupled dimers, a finding which is in line with recent predictions made on the basis of $a b$ initio computer simulations. ${ }^{7}$

The information here provided regarding the spindiffusion coefficient as well as its associated relaxation time tells that characteristic spin-flip times are significantly shorter than those required for large scale molecular reorientations. To put things into context, let us remark that the time scales associated with spin-flip phenomena compare favorably with those reported for the residence time of an $\mathrm{O}_{4}$ $\operatorname{dimer}^{7}$ in the liquid phase. Furthermore, oxygen molecules at the $6 d$ sites in GOX rotate within the planes orthogonal to the chain axis and execute large-amplitude angular excursions $\left(\sim 40^{\circ}\right)$. Also, the value derived for $\tau_{s}$ is also compatible with the time a disk-shaped molecule spends at an equilibrium configuration before jumping to a next site. ${ }^{9}$

The present data can also be compared to $\mu \mathrm{SR}$ measurements of zero-field relaxation rates $\lambda_{\mathrm{ZF}}(T) .{ }^{31}$ These data yield correlation times below 1 ps or so, those for GOX being significantly larger than those found for the liquid. The appearance of a maximum $\lambda_{\mathrm{ZF}}(T)$ at the $\gamma \rightarrow \beta$ transition can thus be explained as follows. On general grounds we can write ${ }^{31}$

$$
\lambda_{\mathrm{ZF}}(T)=2 \gamma_{\mu}^{2} H_{L}^{2} \tau
$$

where $\gamma_{\mu}$ is the muon gyromagnetic ratio, $H_{L}$ is the rootsecond moment of the field distribution at the muon site, and $\tau$ stands for a correlation time which is to be taken as an inverse flipping rate. Within the liquid, the mild temperature dependence of $\lambda_{\mathrm{ZF}}(T)$ arises from extreme motional narrowing effects due to fast roto-translational motions. Spin-flip processes would mostly take place within the lapse of time an $\mathrm{O}_{4}$ dimer may exist, since it involves distances shorter than $3.8 \AA$. Once the GOX crystal is formed, the distance which enables spin flips is kept fixed by the crystal structure and, therefore, the increase in relaxation rate with decreasing temperature up to the $\gamma \rightarrow \beta$ transition is understood as resulting from an increase in the $\tau_{s}$ spin-diffusion relaxation time due to a increase in $J_{n n}$. Within the $\beta$ phase there exist magnetically short-range-ordered (SRO) regions that coexist with a background of paramagnetic excitations. Such shortrange-ordered regions ${ }^{8,32}$ are characterized by an average correlation length

$$
\eta_{\beta} \propto a \sqrt{\exp \left(\pi J_{n n} / \sqrt{3} T\right)}
$$

which is given in terms of the lattice constant $a$ and the nearest-neighbor exchange constant varies from about $3 a$ $\approx 10 \AA$ at $24 \mathrm{~K}$ (just above the $\alpha \rightarrow \beta$ transition) down to some $2 a \approx 6 \AA$ at $43 \mathrm{~K}$ (just below the transition into the $\gamma$ phase), while the corresponding variation in the lattice volume can only account for about $3 \%$ of such a reduction. Also, the number of such regions $n_{\eta}$ is expected to increase as the temperature is decreased. Experimental data, however, show $^{31,33}$ that the quasielastic spectrum does not largely change with temperature and thus one does not expect to find large variations for $D_{s}$ either. The presence of SRO regions provides efficient sinks for muons, which will become trapped within their relatively large fields. The relevant time entering Eq. (15) will now be $\tau_{\mu}=\left(2 \pi \eta_{\beta} n_{\eta} D_{\mu}\right)^{-1}$. Since both $\eta_{\beta}$ and $n_{\eta}$ increase substantially when the temperature is lowered, while the diffusion coefficient is not expected to vary by a large amount, the net result will be a decrease in $\tau_{\mu}$ and thus in $\lambda_{\mathrm{ZF}}(T)$ with decreasing temperature down to a value close to that attained within the magnetically ordered $\alpha$ phase, as it is indeed observed.

To conclude, let us recall that, although liquid oxygen has been under experimental and theoretical scrutinies for about a century, there are a number of issues concerning several of its macroscopic properties which still resist a solid quantitative understanding. Phenomena related to basic thermal and transport properties such as the extremely low value of the vapor pressure at the triple point $(\sim 1.5 \mathrm{mbar})$, its large viscosity if compared to nitrogen, ${ }^{26}$ or the anomalously large decrease with temperature of the thermal conductivity ${ }^{34}$ are all attributed to the presence of magnetic correlations. The way in which such dynamic phenomena give rise to the alluded behaviors is now beginning to be understood on semiquantitative grounds within some theoretical approaches. ${ }^{35}$ These predict the occurrence of measurable effects of the spin degrees of freedom on several quantities, which become well defined only within the hydrodynamic realm such as the sound velocity and sound damping coefficient. Here we have shown that the ultimate microscopic processes involving spin exchange which are at the origin of the observed macroscopic magnetic response of the liquid involve distances comparable to those of closest approach and subpicosecond timescales.

\section{ACKNOWLEDGMENTS}

We thank A. J. Church and C. M. Goodway from the ISIS User Support Group for their expert assistance during the experiments. F.F.A. gratefully acknowledges the financial support from the UK Science and Technology Facilities Council.

\footnotetext{
*Also at Department of Physics and Astronomy, University College London, Gower Street, London WC1E 6BT, United Kingdom; f.fernandez-alonso@rl.ac.uk †javier@langran.iem.csic.es

${ }^{1}$ R. C. Youngquist, C. D. Immer, J. E. Lane, and J. C. Simpson, IEEE Trans. Magn. 39, 2068 (2003); A. T. Catherall, K. A.
}

Benedict, P. J. King, and L. Eaves, Phys. Rev. E 68, 037302 (2003); A. T. Catherall, L. Eaves, P. J. King, and S. R. Booth, Nature (London) 422, 579 (2003); M. Takeda and K. Nishigaki, Phys. Rev. A 43, 2081 (1991).

${ }^{2}$ C. Uyeda, A. Yamagishi, and M. Date, J. Phys. Soc. Jpn. 55, 468 (1986); 56, 3444 (1987). 
${ }^{3}$ F. F. Roberts, Proc. Phys. Soc. London, Sect. B 65, 460 (1952).

${ }^{4}$ K. P. Huber and G. Herzberg, Molecular Spectra and Molecular Structure IV. Constants of Diatomic Molecules (Van Nostrand, New York, 1979).

${ }^{5}$ F. Dunstetter, V. Plakhty, and J. Schweizer, J. Magn. Magn. Mater. 96, 282 (1991).

${ }^{6}$ R. J. Meier, C. J. Schinkel, and A. de Wisser, J. Phys. C 15, 1015 (1982); A. Perrier, Leiden Commun C 139, 25 (1914). A value of $\chi(T=65 \mathrm{~K})=289 \times 10^{-6} \mathrm{~cm}^{3} \mathrm{~g}^{-1}$ has been used for the liquid.

${ }^{7}$ T. Oda and A. Pasquarello, J. Phys.: Condens. Matter 15, S89 (2003); Phys. Rev. B 70, 134402 (2004).

${ }^{8}$ Y. A. Freiman and H. J. Jodl, Phys. Rep. 401, 1 (2004).

${ }^{9}$ F. Dunstetter, O. Hardouin-Duparc, V. P. Plakhty, J. Schweizer, and A. Delapalme, Low Temp. Phys. 22, 101 (1996); F. Dunstetter, Ph.D. thesis, University of Paris-Sud, 1998.

${ }^{10}$ A. de Bernabé, G. J. Cuello, F. J. Bermejo, F. R. Trouw, and A. P. J. Jansen, Phys. Rev. B 58, 14442 (1998).

${ }^{11}$ A. Chahid, M. García-Hernández, F. J. Bermejo, J. L. Martínez, E. Enciso, and F. J. Mompean, Physica B (Amsterdam) 182, 409 (1992); J. L. Martinez, F. J. Bermejo, M. Garcia-Hernandez, and F. J. Mompean, J. Phys.: Condens. Matter 3, 3849 (1991); A. Chahid, F. J. Bermejo, E. Enciso, M. Garcia-Hernandez, and J. L. Martinez, ibid. 5, 423 (1993).

${ }^{12}$ M. Arai, A. D. Taylor, S. M. Bennington, and Z. A. Bowden, in Recent Developments in the Physics of Fluids, edited by W. S. Howells and A. K. Soper (Adam Hilger, Bristol, 1992), p. F291.

${ }^{13}$ I. Bustinduy, F. J. Bermejo, T. G. Perring, and G. Bordel, Nucl. Instrum. Methods Phys. Res. A 546, 498 (2005); Rev. Sci. Instrum. 78, 043901 (2007).

${ }^{14}$ M. Deraman, J. C. Dore, and J. Schweizer, J. Magn. Magn. Mater. 50, 178 (1985).

${ }^{15}$ D. S. Sivia, C. J. Carlile, W. S. Howells, and S. Konig, Physica B 182, 341 (1992); D. Sivia and J. Skilling, Data Analysis: A Bayesian Tutorial (Oxford University Press, Oxford, 2006), Chap. 4.

${ }^{16}$ E. G. D. Cohen, P. Westerhuijs, and I. M. de Schepper, Phys. Rev. Lett. 59, 2872 (1987); For some recent tests, see M. D. Ruiz-Martin, M. Jimenez-Ruiz, M. Plazanet, F. J. Bermejo, R. Fernandez-Perea, and C. Cabrillo, Phys. Rev. B 75, 224202 (2007); C. Mondelli, M. A. González, F. Albergamo, C. Carbajo, M. J. Torralvo, E. Enciso, F. J. Bermejo, R. Fernández-Perea, C. Cabrillo, V. Leon, and M. L. Saboungi, ibid. 73, 094206 (2006); F. J. Bermejo, I. Bustinduy, S. J. Levett, J. W. Taylor, R. Fernandez-Perea, and C. Cabrillo, ibid. 72, 104103 (2005).

${ }^{17}$ B. A. Younglove, J. Phys. Chem. Ref. Data 11, 307 (1982).

${ }^{18}$ H. Kiefte and M. J. Clouter, J. Chem. Phys. 62, 4780 (1975); M.
J. Clouter and H. Kiefte, ibid. 59, 2537 (1973); G. C. Straty and B. A. Younglove, J. Chem. Thermodyn. 5, 305 (1973); A. van Iterbeek and W. van Dael, Physica (Amsterdam) 28, 861 (1962).

${ }^{19}$ P. G. de Gennes, J. Phys. Chem. Solids 4, 223 (1958); S. W. Lovesey, Theory of Neutron Scattering from Condensed Matter (Oxford University Press, Oxford, 1984), Vol. II, p. 325.

${ }^{20}$ C. Uyeda, A. Yamagishi, H. Hori, and M. Date, Physica B (Amsterdam) 155, 421 (1989); J. Phys. Soc. Jpn. 54, 1107 (1985).

${ }^{21}$ P. J. Dunlop and C. M. Bignell, J. Chem. Phys. 108, 7301 (1998).

${ }^{22}$ J. Warnock and D. D. Awschalom, Phys. Rev. B 35, 1962 (1987).

${ }^{23}$ V. F. Sears, Can. J. Phys. 44, 1279 (1966); 44, 1299 (1966).

${ }^{24}$ F. Fernandez-Alonso, F. J. Bermejo, S. E. McLain, J. F. C. Turner, J. J. Molaison, and K. W. Herwig, Phys. Rev. Lett. 98, 077801 (2007).

${ }^{25}$ D. L. Price, M. L. Saboungi, and F. J. Bermejo, Rep. Prog. Phys. 66, 407 (2003).

${ }^{26}$ D. K. Hilton and S. W. Van Sciver, Cryogenics 48, 56 (2008).

${ }^{27}$ G. Müller, Phys. Rev. Lett. 60, 2785 (1988); O. F. de Alcantara Bonfim and G. Reiter, ibid. 69, 367 (1992); N. Srivastava, J. M. Liu, V. S. Viswanath, and G. Müller, J. Appl. Phys. 75, 6751 (1994); I. M. Nolden and R. J. Silbey, Phys. Rev. B 54, 381 (1996).

${ }^{28}$ H. Mori and K. Kawasaki, Prog. Theor. Phys. 27, 529 (1962); H. S. Bennett and P. C. Martin, Phys. Rev. 138, A608 (1965); T. Morita, J. Phys. Soc. Jpn. 39, 1217 (1975); W. Y. Ching, K. M. Leung, and D. L. Huber, Solid State Commun. 24, 457 (1977); B. N. Narozhny, Phys. Rev. B 54, 3311 (1996); D. L. Huber, J. Phys.: Condens. Matter 15, L579 (2003).

${ }^{29}$ P. G. de Gennes, Physica (Amsterdam) 25, 825 (1959).

${ }^{30}$ L. P. Kadanoff and P. C. Martin, Ann. Phys. (N.Y.) 281, 800 (2000).

${ }^{31}$ F. J. Bermejo, S. F. J. Cox, F. J. Mompeán, M. Garcia, M. L. Senent, and J. L. Martinez, Philos. Mag. B 73, 689 (1996); F. J. Bermejo, A. de Bernabé, J. L. Martinez, M. L. Senent, G. J. Cuello, S. F. J. Cox, F. Dunstetter, and F. Trouw, see also J. Low Temp. Phys. 111, 287 (1998).

${ }^{32}$ V. A. Slosarev, Y. A. Freiman, and R. P. Yankelevich, Sov. J. Low Temp. Phys. 6, 105 (1980).

${ }^{33}$ P. W. Stephens, R. J. Birgeneau, C. F. Majkrzak, and G. Shirane, Phys. Rev. B 28, 452 (1983).

${ }^{34}$ D. Celik and S. W. Van Sciver, Cryogenics 45, 620 (2005); T. E. Waterman, D. P. Kirsch, and R. I. Brabets, J. Chem. Phys. 29, 905 (1958); A. C. Jenkins and F. S. DiPaolo, ibid. 25, 296 (1956).

${ }^{35}$ I. M. Mryglod and R. Folk, Physica A 234, 129 (1996). 